\title{
ON BURNER-STABILIZED CYLINDRICAL PREMIXED FLAMES IN MICROGRAVITY
}

\author{
James A. Eng, Delin Zhu and Chung K. Law \\ Department of Mechanical and Aerospace Engineering \\ Princeton University, Princeton, NJ 08544
}

Introduction

A class of one-dimensional premixed flames that has the potential of eliminating heat loss and stretch effects is the burner-generated cylindrical and spherical flames, where a premixed gas flows through a porous cylindrical or spherical burner and the resulting flame is formed at some distance from the bumer, as shown in Figs. la and $1 \mathrm{~b}$. To demonstrate that such flames are curved but not stretched, we consider the general expression for the stretch rate experienced by a flame surface $[1,2]$,

$$
\begin{aligned}
K & =\frac{1}{A} \frac{d A}{d t} \\
& =-n \cdot \nabla \times\left(u_{f} \times n\right)+\left(s_{f} \cdot n\right)(\nabla \cdot n)
\end{aligned}
$$

where $A$ is the area of an infinitesimal element of the flame surface, $u_{f}$ the flow velocity evaluated at the flame, $s_{f}$ the velocity of the flame, and $n$ the unit normal vector of the surface. Equation (1) shows that, since the flame is stationary $\left(s_{\mathrm{f}} \equiv 0\right)$, and the flow is normal to the flame surface $\left(u_{\mathrm{f}} \times \mathbf{n} \equiv 0\right)$, the stretch rate vanishes identically. Furthermore, while such a flame can be stabilized by heat loss to the burner, the divergent nature of the flow provides an additional stabilization mechanism. That is, as the flow rate is continuously increased, the flame recedes from the burner and heat loss can be eventually reduced to become negligible as compared to the heat generated at the flame. At this point the flame temperature is expected to be close to the adiabatic flame temperature. Further increase in the flow rate is then accommodated by an increase in the flame standoff distance to a position where the flame speed again balances the flow velocity. Thus, the cylindrical/spherical geometry offers a unique advantage for the study of adiabatic, one-dimensional, stretch-free flames that is not present in other commonly used experimental systems. Such flames, however, can only be established, and studied, in a microgravity ( $\mu g)$ environment because of the distorting nature of the buoyant flow under normal gravity conditions.

In view of the above considerations, an experimental and theoretical program on cylindrical and spherical premixed flames in microgravity has been initiated. We are especially interested in: (1) assessing heat loss versus flow divergence as the dominant stabilization mechanism; (2) understanding the effects of flame curvature on the burning intensity; and (3) determining the laminar burning velocity by using this configuration. In the present study we have performed analytical, computational, and $\mu \mathrm{g}$-experimental investigations of the cylindrical flame. The results are presented in the following sections.

\section{Asymptotic Analysis and Oualitative Behavior}

The problem analyzed is shown in Fig. 1a. Here a premixture of temperature $T_{\mathfrak{u}}$, mass flow rate $m$, and consisting of a deficient, rate-limiting reactant $F$ (fuel) with mass fraction $Y_{F, u}$ issues from a unit-length, internally-cooled porous cylinder which has a radius $r_{z}$ and surface temperature $T_{z}$, where the subscript $s$ designates the bumer surface. From continuity, the mass flow rate $m=2 \pi r \rho u$ is a conserved quantity along the radial distance $r$, where $u$ is the $r$-velocity. Upon ignition, a cylindrical flame is established concentric with the burner, with the thin reaction zone located at $\mathbf{r}_{f}$ and the preheat zone extending from the burner surface to $r_{f}$, where the subscript $f$ designates the reaction zone. From the experimental point of view, the parameter which can be independently and conveniently controlled is $\mathrm{m}$. Thus for a given $\mathrm{m}$, the cooling rate of the burner and hence the heat loss rate from the flame, $Q_{s}$, can be adjusted to achieve a constant $T_{s}$. The flame temperature $T_{f}$ and flame standoff location $r_{f}$ will also adjust accordingly for the given $m$ and $T_{s}$. 
A straightforward application of activation energy asymptotics yields the following flame responses,

$$
\begin{aligned}
& \left(\frac{\tilde{m}}{\tilde{\mathrm{f}}_{f}}\right)^{2}=\operatorname{Le} \Delta \\
& \tilde{\mathrm{T}}_{\mathrm{f}}=\left(\overline{\mathrm{T}}_{\mathrm{f}}^{0}+\tilde{\mathrm{T}}_{\mathrm{s}}-\tilde{\mathrm{T}}_{\mathrm{u}}\right)-\tilde{\mathrm{q}}_{\mathrm{s}} \\
& \tilde{\mathrm{q}}_{\mathrm{s}}=\tilde{\mathrm{r}}_{\mathrm{f}}^{-\tilde{\mathrm{m}}}
\end{aligned}
$$

where $\Delta\left(\tilde{\mathrm{T}}_{\mathrm{f}}\right)=2 \mathrm{Da}\left(\tilde{\mathrm{T}}_{\mathrm{f}}^{2} / \tilde{\mathrm{T}}_{\mathrm{a}}\right)^{2} \exp \left(-\tilde{\mathrm{T}}_{\S} \tilde{\mathrm{T}}_{\mathrm{f}}\right)$ is a reduced Damköhler number,

$$
\mathrm{Da}=\left(\frac{\mathrm{BY}}{\mathrm{W}_{\mathrm{O}, \mathrm{u}}}\right)\left(\frac{\rho_{\mathrm{b}}^{2} \mathrm{r}_{\mathrm{s}}^{2}}{\lambda / \mathrm{c}_{\mathrm{p}}}\right)
$$

is the Damkohler number, $L$ e the Lewis number, $\tilde{m}=m /\left(2 \pi \lambda / c_{p}\right), \tilde{T}=c_{p} T / Y_{F, u} q_{c}, \bar{Y}_{F}=Y_{F} / Y_{F, u}, \tilde{p}=\rho / p_{b}, \tilde{r}=r / r_{s}$, $\tilde{q}_{s}=\left[r_{s} /\left(Y_{F y} q_{c}\right)\right] q_{s}, T_{a}$ is the activation temperature, $\rho$ the density, $Y_{O}$ the mass fraction of the abundant species, $\lambda$ the thermal conductivity, $c_{p}$ the specific heat, $q_{c}$ the chemical heat release per unit mass of fuel, $q_{z}$ the specific heat loss per unit length of burner, and $B$ the frequency factor. The subscripts $u$ and $b$ respectively designate the unburnt and burnt states of the flame, and the superscript " 0 " designates the adiabatic, one-dimensional planar flame. We shall also assume that there is no downstream heat loss such that $T_{f} \equiv T_{b}$.

For large values of $\tilde{r}_{f}$ the above solution approaches the adiabatic limit, with $\tilde{q}_{k} \rightarrow 0, \tilde{T}_{f} \rightarrow \bar{T}_{f}^{o}$ and $\Delta\left(\tilde{\mathrm{T}}_{\mathrm{f}}\right) \rightarrow \Delta\left(\mathrm{T}_{\mathrm{f}}^{\mathrm{g}}\right)$ which is a constant. The last relation, when interpreted in the context of Eq. (2), implies that $\left(\tilde{\mathrm{m}} / \tilde{\mathrm{r}}_{\mathrm{f}}\right)$ becomes a property of the mixture in this limit, being independent of the burning rate $\bar{m}$ and the flame curvature which is proportional to $\overline{\mathrm{f}}_{\mathrm{f}}^{-1}$. This result has the following implications.

First, since $\tilde{\mathrm{f}}_{\mathrm{f}} \sim \tilde{\mathrm{m}}$, the flame accommodates an increase in the mass flow rate from the burner by moving to a larger radial distance and hence attaining a larger flame surface area. The flame is therefore stabilized through flow divergence. This stabilization mechanism and hence flexibility do not exist for the flat-burner flame, which loses its planar nature when the burner discharge rate exceeds that of the adiabatic valu

Second, if we evaluate $\tilde{m}$ at the reaction zone, then $\left(\tilde{\mathrm{m}} / \tilde{r}_{\mathrm{f}}\right)=\left(\rho_{\mathrm{b}} \mathrm{u}_{\mathrm{f}}\right) /\left(\lambda / \mathrm{c}_{\mathrm{p}}\right)$ is simply a nondimensional mass flux at $\tilde{r}_{f}$, say $\overline{\mathrm{f}}_{f}$, where $\overline{\mathrm{f}}=\mathrm{fr}_{f} /\left(\lambda / c_{p}\right)$ is the nondimensional $f$. Furthermore, since this quantity is independent of $\tilde{\mathrm{r}}_{f}$ and hence the flame curvature in the adiabatic limit, it must be equal to the (constant) mass flux of the adiabatic planar flame, $\tilde{\mathrm{f}}^{\circ}=\widetilde{\mathrm{f}}_{\mathrm{u}}^{\circ}=\widetilde{\mathrm{f}}_{\mathrm{f}}^{\circ}$, for which $\mathrm{r}_{\mathrm{s}}$ can be considered as an arbitrary length for nondimensionalization. We therefore have the following sequence of identities in the adiabatic limit:

$$
\left(\frac{\tilde{\mathrm{m}}}{\tilde{\mathrm{I}}_{\mathrm{f}}}\right)^{2}=\left(\mathrm{f}_{\mathrm{f}}\right)^{2}=\left(\tilde{f}^{0}\right)^{2}=\operatorname{Le} \Delta .
$$

The invariance of $\tilde{f}_{f}$ to flame radius variations provides a simple relation for an experimental determination of the laminar burning velocity of the one-dimensional planar flame. That is, since $m\left(=2 \pi_{f} \rho_{b} u_{f}\right)$ is given while the flame radius can also be easily measured photographically, the laminar burning flux is simply

$$
\mathbf{f}^{\circ}=\frac{\mathbf{m}}{2 \pi r_{\mathrm{f}}}
$$

Furthermore, since $f^{\circ}=f_{u}^{\circ}=\rho_{u} 4_{b^{\circ}}^{\circ}$, the laminar burning velocity is then given by $s_{u}^{o}=u_{u}^{\circ}=f^{\circ} / \rho_{u}$. Our experimental result agrees well with Eq. (7), as will be shown later.

Third, since $f_{f}=f_{u}^{\circ}$ while $f_{f}<f_{u}$ because of flow divergence, we must have $f_{u}^{\circ}<f_{u}$. Thus if $f_{u}$ is identified as the flame propagation rate relative to the freestream, then the concave nature of the present cylindrical flame renders it to have a higher flux and hence propagation rate than the planar flame. This is the same curvature effect which causes the tip of a unity Lewis number Bunsen flame to have a higher burning velocity than its shoulder.

The characteristics of the cylindrical flame can be better demonstrated with the following quantities,

$$
\hat{\mathrm{m}}_{\mathrm{s}}^{2}=\left(\frac{\tilde{\mathrm{m}}}{\tilde{\mathrm{m}}_{\mathrm{s}}^{0}}\right)^{2}=\left(\tilde{\mathrm{T}}_{\mathrm{f}}\right)^{2}\left(\frac{\tilde{\mathrm{T}}_{\mathrm{f}}}{\tilde{\mathrm{T}}_{\mathrm{f}}^{0}}\right)^{4} \exp \left[\tilde{\mathrm{T}}_{\mathrm{a}}\left(\frac{1}{\tilde{\mathrm{T}}_{\mathrm{f}}^{0}}-\frac{1}{\overline{\mathrm{T}}_{\mathrm{f}}}\right)\right]
$$




$$
\hat{\mathbf{Q}}_{\mathrm{s}}=\left(\frac{\tilde{\mathrm{m}}}{\tilde{\mathrm{m}}_{\mathrm{s}}^{\circ}}\right) \tilde{\mathrm{q}}_{\mathrm{s}}=\hat{\mathrm{m}}_{\mathrm{s}} \overline{\mathrm{f}}_{\mathrm{f}}^{-\tilde{\mathrm{m}}}
$$

where $\hat{\mathrm{m}}_{\mathrm{s}}$ is the ratio of the mass burning rate of the cylindrical flame to that of the adiabatic planar flame with the same cylinder surface area, or $m_{s}^{\circ}=2 \pi{ }_{g} s_{s}^{\circ}$. We also define a flame standoff distance $d_{f}=r_{f}-r_{z}$, with $r_{s} \equiv 0$ for the planar case, and nondimensionalize it by the laminar flame thickness $\delta^{\circ}=\left(\lambda / c_{p}\right) / f^{\circ}$ such that $d_{f}=d_{f} / \delta^{\circ}$.

For comparison, we note that for the nonadiabadic planar flame stabilized over a flat burner $[3,4,5]$, $\hat{\mathrm{m}}_{\mathrm{s}}=\hat{\mathrm{f}}=\mathrm{f} / \mathrm{f}^{\circ}$ such that

$$
\begin{aligned}
& \hat{\mathrm{m}}_{\mathrm{s}}^{2}=\left(\frac{\overline{\mathrm{T}}_{\mathrm{f}}}{\overline{\mathrm{T}}_{\mathrm{f}}^{0}}\right)^{4} \exp \left[\tilde{\mathrm{T}}_{\mathrm{s}}\left(\frac{1}{\tilde{\mathrm{T}}_{\mathrm{f}}^{0}}-\frac{1}{\overline{\mathrm{T}}_{\mathrm{f}}}\right)\right] \\
& \hat{\mathrm{Q}}_{\mathrm{s}}=\hat{\mathrm{m}}_{\mathrm{s}} \exp \left(-\hat{\mathrm{m}}_{\mathrm{s}} \hat{\mathrm{d}}_{\mathrm{f}}\right)
\end{aligned}
$$

while Eq. (3) still holds for the relation between $\tilde{\mathrm{T}}_{\mathrm{f}}$ and $\tilde{\mathrm{q}}_{\mathrm{s}}$

Calculated results using representative values show that both the planar and cylindrical cases yield a dual solution behavior in that there are two values of the mass burming rate $\hat{\mathbf{m}}_{\mathbf{g}}$ for either a given heat loss rate $\hat{\mathbf{Q}}_{\mathbf{g}}$ or a given flame standoff distance $\hat{d}_{f}$ The behavior for the planar case is a well known observation $[6,7,8]$ which has also been recently explained $[4,5]$ by noting that by taking $\hat{m}$ as the independent parameter with $T_{s}$ fixed, the flame responses in terms of $\hat{Q}_{s}$ and $\hat{d}_{f}$ are actually unique. By similar reasoning we then expect that the responses of the cylindrical flame are also unique when $\hat{\mathbf{m}}_{s}$ is the independent parameter.

For the cylindrical flame, it is further found that when the cylinder radius is large, the flame behavior follows closely that of the planar flame up to $\hat{\mathrm{m}}_{3} \approx 1$, as expected. In this regime heat loss is the dominant flame stabilization mechanism. For $\hat{m}_{3}>1$, the planar flame does not exist while the cylindrical flame can still be maintained due to flow divergence. In this regime the flame is practically adiabatic, with $\hat{Q}_{5} \approx 0$ and $\bar{T}_{f} \approx \tilde{T}_{f}^{\circ}$. For smaller cylinder radius, the response of the cylindrical flame deviates significantly from that of the planar flame. The flame is almost adiabatic and is stabilized by flow divergence at higher values of $\hat{\mathbf{m}}_{z}$.

To identify the influence of curvature on the burning flux at the upstream boundary of the flame, we have also computed $\hat{\mathrm{f}}_{\mathrm{u}}=\mathrm{f}_{\mathrm{u}} / \mathrm{f}_{\mathrm{a}}^{0}$, with the unburnt state evaluated at the location of the $1 \%$ temperature rise from the freestream temperature according to the suggestion of Tien and Matalon [9]. The results are quite insensitive to the actual state selected as long as it is close to the ambient state. It is found that with increasing $\hat{\mathrm{m}}_{s}, \hat{\mathrm{f}}_{\mathrm{u}}$ initially increases to values of $\hat{f}_{u}>1$. It then decreases and eventually approaches the adiabatic, planar case of $\hat{f}_{u}=1$ as $\hat{\mathrm{f}}_{\mathrm{f}} \rightarrow \infty$. The important point to note is that $f_{u}$ can exceed $f_{a}{ }^{\circ}$, in agreement with the previous discussion of curvature-enhanced burning flux and hence burning velocity.

\section{Microgravity Experimentation}

The experimental apparatus for the cylindrical flame is shown in Fig. 2. The cylindrical burner was made of a porous bronze tube with $5 \mu \mathrm{m}$ pore size, $1.25 \mathrm{~cm}$ diameter, and $3.76 \mathrm{~cm}$ active length. The thickness of the porous wall is $4.7 \mathrm{~mm}$. Since uniformity of the flow through the porous wall depends on the pressure differential across it, the reactant mixture was supplied from both ends of the burner tube so as to minimize the internal pressure gradient in the axial direction of the cylinder. Furthermore, stainless steel tubes with small holes at various locations were positioned inside the burner to further even the axial pressure distribution. A cross sectional view of the burner with the internal tubes is shown in the inset of Fig. 2. The axial pressure distribution was measured (under normal gravity conditions) by drilling small holes in the porous wall and connecting water manometers to the pressure taps. For the best achieved design of the number and placement of the holes in internal tubes at a typical flow rate, the inlet gage pressure to the burner was $60 \mathrm{~cm}$ water and the axial pressure variation was $+1-2 \mathrm{~cm}$ water, a $10 \%$ variation.

Due to the small burner size and the limited duration of the microgravity experiments, no cooling was used for the burner. A thermocouple was inserted inside the burner wall to record the burner temperature. A hot wire mounted on a rotary solenoid was used to ignite the mixture. Experiments were performed under microgravity conditions at the NASA-Lewis 2.2 second drop tower. Mass flow rates were measured by using sonic flow nozzles whose upstream pressures were recorded by using pressure transducers. A video camera was used to record the flame shape and standoff distance. 
Computational Simulation

The experimental situation was numerically simulated by using the Sandia premixed flame code [10], with modifications to ensure strict mass conservation in the divergent flow field. The program solves the governing equations of mass, energy and species using finite difference approximation, allowing for detailed chemical kinetics, multicomponent diffusion and variable thermal properties. The chemical mechanism used was that developed by Egolfopoulos et al. [11], which has 30 species and 153 reaction steps.

The independent variable for the burner stabilized flame is the mass flow rate $m$. The necessary boundary conditions are the burner surface temperature, $T_{3}$, and the reactant mixture mass flux fractions. The boundary conditions imposed on the product side are vanishing gradients for species and temperature, $d Y_{j} / d r$ and dT/dr. The program solves the governing equations to determine the temperature profile through the flame, from which the flame location and heat loss to the burner can be found.

\section{Experimental and Computational Results}

A mixture of $\mathrm{CH}_{4}+2 \mathrm{O}_{2}+7.52 \mathrm{~N}_{2}+5.665 \mathrm{He}$, with an effective Lewis number of 1.88 , was used for the cylindrical flame experiments. Helium was added to enhance flamefront stability. There are two causes for the enhanced flame stability with helium addition. First, the effective Lewis number of the mixture increases, which reduces the thermal-diffusional instability. Second, the adiabatic flame temperature is reduced, which reduces the density jump across the flame and the propensity to develop the hydrodynamic (Landau) instability. For this mixture the adiabatic flame temperature for the unburned mixture temperature of $300 \mathrm{~K}$ is $1885 \mathrm{~K}$, and the calculated laminar flame speed is $20.8 \mathrm{~cm} / \mathrm{s}$, which corresponds to a mass burning flux of $0.016 \mathrm{gm} / \mathrm{cm}^{2}-\mathrm{s}$.

Figure 3 compares the calculated and measured flame standoff distance as a function of the normalized mass flow $\hat{\mathrm{m}}_{\mathbf{s}}$. The inset shows a typical recorded flame configuration, which exhibits some large-scale, weak wrinkling. This wrinkling is believed to be caused by the pressure variation in the burner and leads to a variable standoff distance of $+/-15 \%$ in the axial direction. The spread in the experimental data therefore represents the extent of flame nonuniformity as measured by the mimimum and maximum standoff distances. The flame location for the numerical results is taken as that of the maximum $\mathrm{CH}$ radical concentration, since this species is chiefly responsible for the luminosity of the flame and assumes its maximum concentration in the active reaction zone of the flame. The heat loss to the burner is also presented for the calculated results, and shows that around a normalized flow rate of $\hat{\mathrm{m}}_{\mathrm{s}} \sim 1.4$, the heat loss is essentially zero. At this point the stabilization mechanism has changed from heat loss to flow divergence. The figure also shows that all the microgravity data are in the regime where the flame is stabilized by flow divergence. This is further substantiated by the experimental result that the thermocouple temperature basically remained at the ambient value during the experiment. The comparison shows that the flame is situated further away than calculated, with about 1 to $2 \mathrm{~mm}$ differences. Overall, the experimental and computational results agree quite well, at least qualitatively, considering the difficulties usually associated with microgravity experimentation.

Figure 4 compares the calculated and observed mass flux through the reaction zone. As discussed, the calculated result in the adiabatic limit should be close to the independently calculated laminar burning flux for the adiabatic planar flame. Exact agreement is not expected because while the calculated result is a constant for the planar flame, it depends on the present choice of the maximum $\mathrm{CH}$ radical location as that of the reaction zone for the cylindrical flame. The uncertainty, however, is not expected to be large because the flame thickness is much smaller than its standoff distance. It is seen that the calculated laminar mass flux attains a value of about $f_{f}=0.0156$ $\mathrm{gm} / \mathrm{cm}^{2}-\mathrm{s}$ in the divergence-stabilized regime, which compares very favorably with the independently calculated value of $f^{\circ}=0.016 \mathrm{gm} / \mathrm{cm}^{2}-\mathrm{s}$ for the adiabatic planar flame. Figure 4 further shows that the experimentally measured values also agree well with the computed results, being slightly smaller. This demonstrates the feasibility of determining the laminar burning flux, and hence the laminar buming velocity, by using the cylindrical/spherical flame as proposed herein.

The difference between the calculated and measured values for the flame location and burning flux can be attributed to the small influence of gas-phase heat loss in the experiment, which is not included in the calculations. Perhaps most importantly, while the calculation assumes an adiabatic downstream, in the experiments the downstream temperature is that of the ambience. This downstream heat loss reduces the flame burning rate, which in turn causes the slightly weakened flame to move to a larger radial location so that stabilization is achieved with the reduced radial velocity. 


\section{Concluding Remarks}

In the present investigation we have demonstrated the usefulness of the stationary cylindrical flame for fundamental flame studies, especially when the flame is stabilized by flow divergence and hence is basically adiabatic. Since these curved flames are stretchless, the influence of curvature on the flame structure and response can be unambiguously studied. Specifically, we have shown that the downstream burning flux of such flames are unaffected by the flame curvature and hence is equal to the constant burning flux of the adiabatic freely-propagating planar flame, while the upstream burning flux exceeds it due to flow divergence. This understanding is useful to the study of the general nature of stretched flames subjected to the influence of flame curvature, especially within the context of laminar flamelets in the modeling of turbulent flames.

We have also successfully produced a nearly-cylindrical and adiabatic flame in the microgravity environment of the drop tower. While the quality of the flame still needs to be improved, the viability of the concept is nevertheless established, especially in light of the favorable agreement between the microgravity results and detailed numerical computation.

A further contribution of the present study is the possibility of determining the laminar burning velocities of combustible mixtures by using the cylindrical flame in the nearly-adiabatic regime. While microgravity experiments are fairly complex and possibly also costly in terms of their operation, the simplicity of the experimental configuration and concept renders this methodology a useful substantiating alternative to other approaches for such a determination, at least for reasonably smooth flames.

Finally, we do wish to emphasize again the difficulty in obtaining perfectly cylindrical flames without wrinkles, even with the addition of helium. If such wrinkles are inherent to the flame, as manifestations of some hydrodynamic instabilities [12], then there would exist a limitation on the quantitative accuracy in determining the flame responses such as the burning rate of practical combustion mixtures. Studies are currently underway to assess the nature of such wrinkles. Experiments will also be conducted with spherical flames; it is anticipated that the additional curvature could partially "absorb" the extent of wrinkling.

\section{References}

1. Law, C. K., Twenty-Second Symposium (International) on Combustion, The Combustion Institute, Pittsburgh, 1988, pp. 1381-1402.

2. Matalon, M., Combust. Sci. Tech. 31: 169-181 (1983).

3. Margolis, S. B., Combust. Sci. Tech. 22: 143-169 (1980).

4. Chao, B. H. and Law, C. K., Combust. Sci. Tech. 62: 211-237 (1988).

5. Eng, J. A., Zhu, D. L. and Law, C. K., "On the Structure, Stabilization, and Dual Response of Flat-Burner Flames," Combust. Flame, in press.

6. Spalding, D. B. and Yumlu, V. S., Combust. Flame 3: 553-556 (1959).

7. Ferguson, C. R. and Keck, J. C., Combust. Flame 34: 85-98 (1979).

8. Yamazaki, S. and Ikai, S., Trans. Japan Soc. Mech. Engrs. 37. 293: 121-130 (1971).

9. Tien, J. H. and Matalon, M., Combust. Flame 84: 238-248 (1991).

10. Kee, R. J., Grear, J. F., Smooke, M. D. and Miller, J. A., A Fortran Program for Modeling Steaty Laminar One-Dimensional Premixed Flames, Sandia National Laboratories Report No. SAND85-8240, 1985.

11. Egolfopoulos, F. N., Du, D. X. and Law, C. K., Combust. Sci. Tech. 83: 33-75 (1992).

12. McIntosh, A C., J. Fluid Mech. 161: 43-75 (1985). 


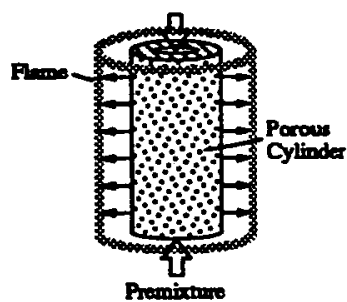

a) Cylindrical Burner Flame

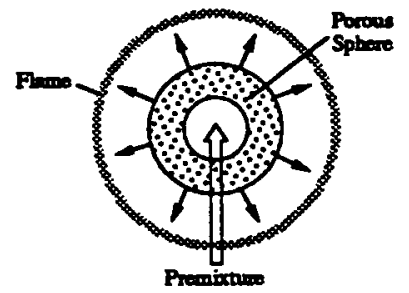

b) Spherical Burner Flame
Figure 1. Configurations of (a) cylindrical, and (b) spherical flames which are curved but unstretched.

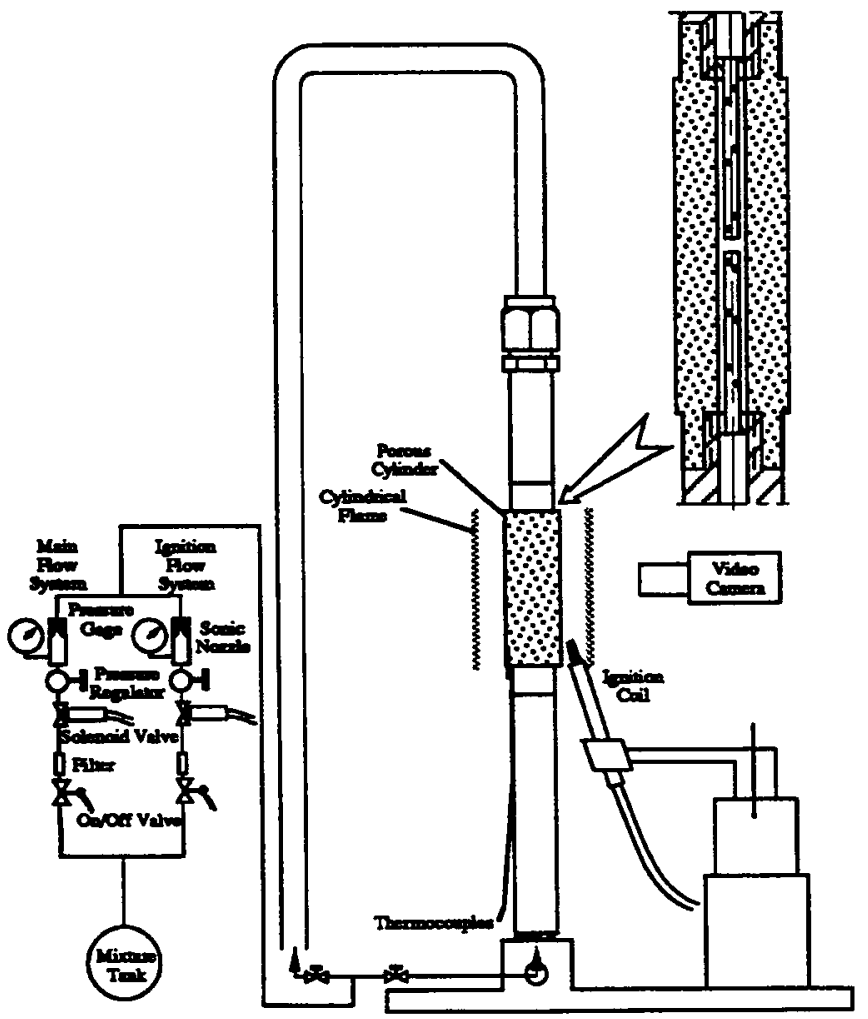

Figure 2. Schematic of the experimental apparatus. Inset is the cross-section of the cylindrical burner.

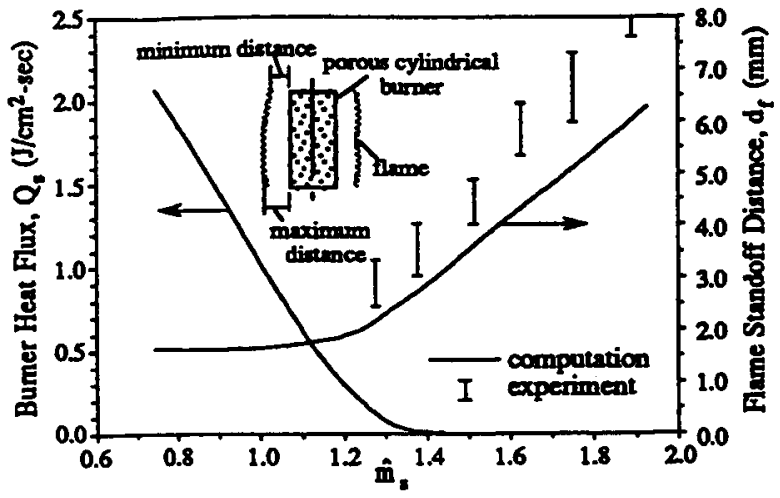

Figure 3. Computed heat loss flux and flame standoff distance, and experimental flame standoff distance, as functions of the discharge rate. Inset is a representative experimental flame configuration.

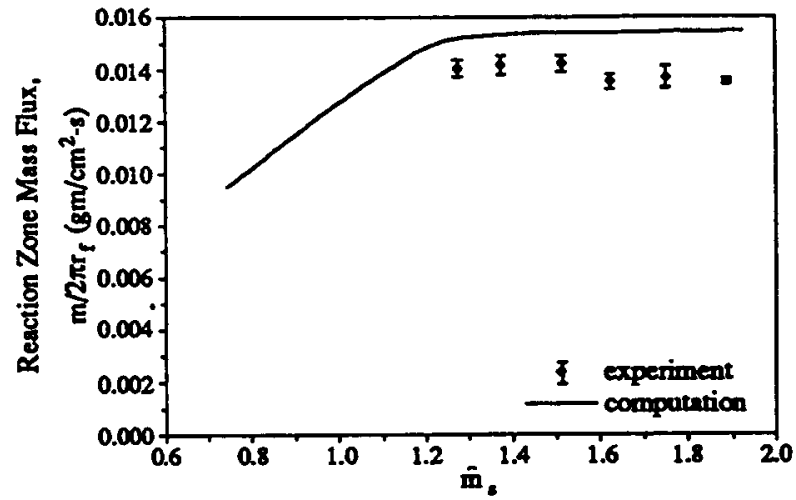

Figure 4. Computed and reaction zone mass flux as function of the discharge rate. 